\title{
Current Trends in Mild Traumatic Brain Injury
}

Received 09/11/2021 Review began 09/19/2021 Review ended 09/29/2021 Published 10/02/2021

\section{(c) Copyright 2021}

Krueger et al. This is an open access article distributed under the terms of the Creative Commons Attribution License CC-BY 4.0., which permits unrestricted use, distribution, and reproduction in any medium, provided the original author and source are credited.

\author{
Evan M. Krueger ${ }^{1}$, Anthony M. DiGiorgio ${ }^{2}$, Jonathan Jagid ${ }^{3}$, Joacir G. Cordeiro ${ }^{3}$, Hamad Farhat ${ }^{4}$
}

1. Neurological Surgery, Carle Foundation Hospital, Urbana, USA 2. Neurological Surgery, University of California San Francisco, San Francisco, USA 3. Neurological Surgery, University of Miami, Coral Gables, USA 4. Neurological Surgery, Advocate Aurora Health Care, Downers Grove, USA

Corresponding author: Evan M. Krueger, dr.evankrueger@gmail.com

\begin{abstract}
In this review, we provide an overview of the current research and treatment of all types of traumatic brain injury (TBI) before illustrating the need for improved care specific to mild TBI patients. Contemporary issues pertaining to acute care of mild TBI including prognostication, neurosurgical intervention, repeat radiographic imaging, reversal of antiplatelet and anticoagulation medications, and cost savings initiatives are reviewed. Lastly, the effect of COVID-19 on TBI is addressed.
\end{abstract}

Categories: Neurosurgery, Trauma

Keywords: covid-19, prognosis, concussion, hemorrhage, mild traumatic brain injury

\section{Introduction And Background}

Mild traumatic brain injury (TBI) is now commonly defined as a traumatic injury presenting a Glasgow coma scale (GCS) score of 13-15, although loss of consciousness and prior to admission amnesia are sometimes still described [1]. This term is often used interchangeably with 'concussion.' However, designating this as 'mild' may be a misnomer. As research on TBI continues to evolve, it is becoming clear that even mild TBI can have significant, life-altering consequences. A prospective analysis out of Transforming Research and Clinical Knowledge in TBI (TRACK-TBI) showed that 12 months after a mild TBI, 53\% of patients reported significant functional limitations, defined as a Glasgow outcome scale extended (GOSE) scores $<8$ [2].

\section{Review}

\section{Current guidelines and research in all TBI}

Professional organizations such as the American Association of Neurologic Surgeons and Congress of Neurologic Surgeons Section of Neurotrauma and Critical Care, National Health Institute funded TBI databases TRACK-TBI, and public outreach programs such as the ThinkFirst Foundation are at the forefront of improving outcomes for TBI patients. Their efforts have translated into decreased TBI mortality rates over the past decade in the United States [3]. Recently it was reported $75 \%$ of moderate TBI patients achieve GOSE scores $\geqslant 4$ at 12 months, and $57 \%$ of severe TBI patients achieve GOSE scores $\geqslant 5$ at six months $[4,5]$. For clinicians, the Brain Trauma Foundation Guidelines for the Management of Severe TBI is an invaluable resource [6]. Recent updates to algorithms and approaches to the management of elevated intracranial pressure and decompressive hemicraniectomy have helped fill the gap between evidence and clinical practice $[7,8]$.

New areas of research will potentially change the management of TBI. These include biomarkers, treatment techniques, and medications. The 2018 A Prospective Clinical Evaluation of Biomarkers of Traumatic Brain Injury (ALERT-TBI) trial led to the first Food and Drug Administration (FDA) approved biomarkers for TBI such as ubiquitin C-terminal hydrolase-L1 (UCH-L1) and glial fibrillary acidic protein (GFAP) [9]. These results are promising and translational research is continuing to determine their optimal clinical usage $[10$, 11]. Abbott Diagnostic is now partnering with the United States Department of Defense and the TRACK-TBI consortium to conduct a multicenter, pivotal clinical trial on the i-STAT Point-of-Care version of UCHL1/GFAP tandem plasma test for mild TBI [12]. Recognition and treatment of post-traumatic cerebral vasospasm appear to be important for secondary injury prevention [13, 14]. Middle meningeal artery embolization may represent a minimally invasive method to treat and prevent the recurrence of chronic subdural hematomas [14, 15]. The Clinical Randomisation of an Antifibrinolytic in Significant Head injury-3 (CRASH-3) trial examined prehospital tranexamic acid (TXA) for TBI, finding a risk reduction for mortality with TXA compared to placebo [16]. However, $35.8 \%$ of the study sample was from a single country, and head injury-related mortality was $6.6 \%$ for patients with GCS 9-15; thus the trial remains controversial [17]. Results from the Randomised Evaluation of Surgery with Craniectomy for patients Undergoing Evacuation of Acute Subdural Haematoma (RESCUE-ASDH) trial that examines clinical outcomes and cost-effectiveness between craniectomy versus craniotomy for acute subdural hematomas, and the results from the Brain Oxygen Optimization in Severe TBI, Phase 3 (BOOST3) trial that compares the safety and efficacy of treatment strategy guided by brain tissue oxygen monitoring and intracranial pressure measurements versus intracranial pressure measurements alone, are both eagerly awaited [18, 19]. 


\section{Issues in mild TBI}

Relatively few guidelines exist for hospital management of mild TBI despite that it accounts for 95\% of all head injuries [20]. This has translated into variable acute care for mild TBI patients [21]. Furthermore, a large percentage of mild TBI patients do not receive adequate discharge instructions or follow-up [22]. Hence there is currently a tremendous opportunity and need for neurosurgeons to spearhead the improvement in the care of mild TBI patients.

Recent attention has been given to simple clinical measures with prognostic value to help guide clinical follow-up. For computed tomography (CT) positive mild TBI patients, intraventricular and/or petechial hemorrhages are particularly associated with worse long-term outcomes (GOSE <5, OR: 3.47), while epidural hematomas are not [23]. Pre-existing psychiatric conditions are risk factors for worse functional outcomes, increased incidence of post-injury posttraumatic stress disorder and major depressive disorder, and suicidal ideation [24-26]. Patients may need long-term, targeted, and pro-active rehabilitative services. It was recently shown that three-month return to work status and post-concussion symptoms are predictive of sixmonth return to work status [27].

The need for neurosurgical intervention in mild TBI patients is generally uncommon. In 2019, a large, single-center retrospective review reported that, in isolated CT positive mild TBI patients, overall neurosurgical intervention rates were 9.4\% [28]. This was higher than the reported rate of 3.5\% from a previous meta-analysis published in 2018 for non-isolated CT positive mild TBI patients [29]. Delayed deterioration necessitating neurosurgical intervention on initially non-surgical CT positive mild TBI patients is a feared complication that often influences decisions to escalate care; however, the incidence of this deterioration has been reported to range from 1.4-1.5\% [28, 30]. Deterioration is reported to be more common in the elderly; potentially due to the larger subdural space and delayed hyperemia and hyperperfusion [31]. Prevalent usage of anticoagulant and antiplatelet medications in the elderly was commonly deemed as culprits for this deterioration, but current evidence suggests otherwise [32, 33]. Reasons for these conflicting data remain unclear.

Pooled data has reported that isolated traumatic subarachnoid hemorrhage to have an even lower, $0.0017 \%$ chance of neurosurgical intervention [34]. This has led some to propose the mere presence of intracranial hemorrhage, in itself, is not sufficient to warrant interhospital transfer [35]. Therefore telemedicine, allowing consulting neurosurgeons to discuss a patient and directly review images, remains an important and evolving aspect of mild TBI care.

Another controversial point that has received attention in recent literature is the role of repeat CT scans in CT positive mild TBI patients. Krueger et al. reported that in CT positive mild TBI patients, only $11.2 \%$ of patients had a worsened repeat CT and that the clinical neurologic exam was the more sensitive and specific way to monitor patients [28]. A 2013 meta-analysis concluded a repeat CT is unnecessary in mild TBI patients who are stable or clinically improving [36]. The appropriate duration of neurologic exam monitoring to prevent morbidity still remains uncertain. Nonetheless, a repeat CT may serve utility in terms of providing a clearly defined uniform hospital policy, medical-legal protection, justification for intrahospital transfer to lower financial cost units or discharge, or justification for resuming anticoagulation medication. The decision to repeat imaging in mild TBI patients remains at the discretion of the individual treating physicians.

The optimal management for CT positive TBI patients taking oral antiplatelet and anticoagulation medication is not clear. Conflicting evidence surrounds the utility of platelet transfusions for patients taking pre-injury antiplatelet medications $[37,38]$. Vitamin $\mathrm{K}$ antagonists appear to be associated with worse outcomes compared to other direct oral anticoagulants, despite that vitamin $\mathrm{K}$ antagonists more commonly receive reversal agents [39]. If given appropriate reversal agents, the use of preoperative anticoagulant medication does not result in increased postoperative bleeding risk in emergent surgical TBI patients [40]. Proving clinical equipoise in high risk, emergency situations is difficult. To our knowledge, no randomized control trials have addressed antiplatelet or anticoagulants reversal agents in surgical or non-surgical CT positive mild TBI patients. Unless contrary robust data becomes available, judicious use of reversal agents will likely remain common practice.

Clearly identifying low-risk mild TBI patients early in triage may be an important branch point during decision making, and represent an opportunity to improve care and lower costs. A single-center, prospective study by Stippler et al. reported a safe, $71 \%$ reduction in CT imaging by allocating CT positive mild TBI patients who were not taking anticoagulants or antiplatelets and without active epidural or subdural hemorrhages $>1 \mathrm{~cm}$, to receive six hours of close neurologic observation only. There were no missed injuries or delayed surgery using this protocol [41]. Yun et al. designed a single-center, prospective study for low-risk CT positive mild TBI patients who were seen in the emergency department that showed safety, efficacy, and reduced admission rates [42]. Another prospective study showed no differences in six-month outcomes for mild TBI patients admitted to various levels of care [43]. In a controversial article, a prospective study proposed a protocol excluding neurosurgeons in the acute management of nonsurgical TBI as a way to reduce cost [44]. A statement from the American Association of Neurologic Surgeons and Congress of Neurologic Surgeons Section of Neurotrauma and Critical Care argued for maintaining neurosurgical 
involvement in TBI [45]. We strongly reaffirm their statement, recognize neurosurgeons' unique training in all nuances of TBI care, and support their role in caring for the entire spectrum of TBI patients.

\section{COVID-19 and TBI}

The COVID-19 pandemic has brought forward unique challenges in managing TBI [46]. The virus appears to affect the central nervous system, but much remains to be learned [47]. Fortunately, emergent operative cases are still being prioritized. However, neurosurgeons are having to adapt and develop novel case triage algorithms [48]. Neurosurgeons may also be forced to consider the appropriate hospital disposition of lowrisk CT positive mild TBI patients in lieu of limited availability of intensive care unit beds. These issues have brought renewed focus to the neurosurgeon's role in the neurointensive care unit. Some have proposed the creation of a new subspecialty, acute care neurosurgery, to better integrate critical care into modern neurosurgery and allow neurosurgeons to focus specifically on emergencies such as TBI [49].

\section{Conclusions}

Care for mild TBI continues to evolve, and hinges on high-quality translational research. Challenges remain in improving outcomes, lowering costs, and improving efficiency. Future evidence-based care guidelines for mild TBI patients can help achieve these goals.

\section{Additional Information \\ Disclosures}

Conflicts of interest: In compliance with the ICMJE uniform disclosure form, all authors declare the following: Payment/services info: All authors have declared that no financial support was received from any organization for the submitted work. Financial relationships: All authors have declared that they have no financial relationships at present or within the previous three years with any organizations that might have an interest in the submitted work. Other relationships: All authors have declared that there are no other relationships or activities that could appear to have influenced the submitted work.

\section{References}

1. Yue JK, Vassar MJ, Lingsma HF, et al.: Transforming research and clinical knowledge in traumatic brain injury pilot: multicenter implementation of the common data elements for traumatic brain injury. J Neurotrauma. 2013, 30:1831-1844. 10.1089/neu.2013.2970

2. Nelson LD, Temkin NR, Dikmen S, et al.: Recovery after mild traumatic brain injury in patients presenting to us level I trauma centers: a transforming research and clinical knowledge in traumatic brain injury (TRACK-TBI) study. JAMA Neurol. 2019, 76:1049-1059. 10.1001/jamaneurol.2019.1313

3. Rates of TBI-related emergency department visits, hospitalizations, and deaths - United States, 2001-2010. (2016). Accessed: August 18, 2020: https://www.cdc.gov/traumaticbraininjury/data/rates.html.

4. Puffer RC, Yue JK, Mesley M, et al.: Recovery trajectories and long-term outcomes in traumatic brain injury: a secondary analysis of the phase 3 citicoline brain injury treatment clinical trial. World Neurosurg. 2019, 125:e909-e915. 10.1016/j.wneu.2019.01.207

5. McCrea MA, Giacino JT, Barber J, et al.: Functional outcomes over the first year after moderate to severe traumatic brain injury in the prospective, longitudinal TRACK-TBI study. JAMA Neurol. 2021, 78:982-992. 10.1001/jamaneurol.2021.2043

6. Carney N, Totten AM, O'Reilly C, et al.: Guidelines for the management of severe traumatic brain injury, fourth edition. Neurosurgery. 2017, 80:6-15. 10.1227/NEU.0000000000001432

7. Hawryluk GW, Aguilera S, Buki A, et al.: A management algorithm for patients with intracranial pressure monitoring: the Seattle International Severe Traumatic Brain Injury Consensus Conference (SIBICC). Intensive Care Med. 2019, 45:1783-1794. 10.1007/s00134-019-05805-9

8. Hawryluk GW, Rubiano AM, Totten AM, et al.: Guidelines for the management of severe traumatic brain injury: 2020 update of the decompressive craniectomy recommendations. Neurosurgery. 2020, 87:427-434. 10.1093/neuros/nyaa278

9. Bazarian JJ, Biberthaler P, Welch RD, et al.: Serum GFAP and UCH-L1 for prediction of absence of intracranial injuries on head CT (ALERT-TBI): a multicentre observational study. Lancet Neurol. 2018, 17:782-789. 10.1016/s1474-4422(18)30231-x

10. Huie JR, Diaz-Arrastia R, Yue JK, et al.: Testing a multivariate proteomic panel for traumatic brain Injury biomarker discovery: a TRACK-TBI pilot study. J Neurotrauma. 2019, 36:100-110. 10.1089/neu.2017.5449

11. Okonkwo DO, Puffer RC, Puccio AM, et al.: Point-of-care platform blood biomarker testing of glial fibrillary acidic protein versus $\mathrm{S} 100$ calcium-binding protein $\mathrm{b}$ for prediction of traumatic brain injuries: $\mathrm{a}$ transforming research and clinical knowledge in traumatic brain injury study. J Neurotrauma. 2020, 37:24602467. 10.1089/neu.2020.7140

12. Wang KK, Kobeissy FH, Shakkour Z, Tyndall JA: Thorough overview of ubiquitin C-terminal hydrolase-L1 and glial fibrillary acidic protein as tandem biomarkers recently cleared by US Food and Drug Administration for the evaluation of intracranial injuries among patients with traumatic brain injury. Acute Med Surg. 2021, 8:e622. 10.1002/ams2.622

13. Al-Mufti F, Amuluru K, Changa A, et al.: Traumatic brain injury and intracranial hemorrhage-induced cerebral vasospasm: a systematic review. Neurosurg Focus. 2017, 43:E14. 10.3171/2017.8.FOCUS17431

14. Kan P, Maragkos GA, Srivatsan A, et al.: Middle meningeal artery embolization for chronic subdural hematoma: a multi-center experience of 154 consecutive embolizations. Neurosurgery. 2021, 88:268-277. 10.1093/neuros/nyaa379 
15. Link TW, Boddu S, Paine SM, Kamel H, Knopman J: Middle meningeal artery embolization for chronic subdural hematoma: a series of 60 cases. Neurosurgery. 2019, 85:801-807. 10.1093/neuros/nyy521

16. Roberts I, Shakur-Still H, Aeron-Thomas A, et al.: Effects of tranexamic acid on death, disability, vascular occlusive events and other morbidities in patients with acute traumatic brain injury (CRASH-3): a randomised, placebo-controlled trial. Lancet. 2019, 394:1713-1723. 10.1016/S0140-6736(19)32233-0

17. Cap AP: CRASH 3: a win for patients with traumatic brain injury . Lancet. 2019, 394:1687-1688. 10.1016/s0140-6736(19)32312-8

18. Protocol 14PRT/6944. (2014). Accessed: August 18, 2020: https://www.thelancet.com/protocolreviews/14PRT-6944.

19. Brain oxygen optimization in severe TBI, phase 3 - full text view . (2018). Accessed: August 18, 2020: https://clinicaltrials.gov/ct2/show/NCT03754114.

20. Report to Congress on traumatic brain injury epidemiology and rehabilitation | concussion | traumatic brain injury | CDC Injury Center. (2015). Accessed: August 18, 2020: https://www.cdc.gov/traumaticbraininjury/pubs/congress_epi_rehab.html.

21. Nishijima DK, Haukoos JS, Newgard CD, et al.: Variability of ICU use in adult patients with minor traumatic intracranial hemorrhage. Ann Emerg Med. 2013, 61:509-517.e4. 10.1016/j.annemergmed.2012.08.024

22. Seabury SA, Gaudette É, Goldman DP, et al.: Assessment of follow-up care after emergency department presentation for mild traumatic brain injury and concussion: results from the TRACK-TBI study. JAMA Netw Open. 2018, 1:e180210. 10.1001/jamanetworkopen.2018.0210

23. Yuh EL, Jain S, Sun X, et al.: Pathological computed tomography features associated with adverse outcomes after mild traumatic brain injury: a TRACK-TBI study with external validation in CENTER-TBI. JAMA Neurol. 2021, 78:1137-1148. 10.1001/jamaneurol.2021.2120

24. Yue JK, Cnossen MC, Winkler EA, et al.: Pre-injury comorbidities are associated with functional impairment and post-concussive symptoms at 3- and 6-months after mild traumatic brain injury: a TRACK-TBI study. Front Neurol. 2019, 10:343. 10.3389/fneur.2019.00343

25. Stein MB, Jain S, Giacino JT, et al.: Risk of posttraumatic stress disorder and major depression in civilian patients after mild traumatic brain injury: a TRACK-TBI study. JAMA Psychiatry. 2019, 76:249-258. 10.1001/jamapsychiatry.2018.4288

26. Campbell-Sills L, Jain S, Sun X, et al.: Risk factors for suicidal ideation following mild traumatic brain injury: a TRACK-TBI study. J Head Trauma Rehabil. 2021, 36:E30-E39. 10.1097/HTR.0000000000000602

27. Yue JK, Phelps RR, Hemmerle DD, et al.: Predictors of six-month inability to return to work in previously employed subjects after mild traumatic brain injury: a TRACK-TBI pilot study. J Concussion. 2021, 5:10.1177/20597002211007271

28. Krueger EM, Putty M, Young M, Gaynor B, Omi E, Farhat H: Neurosurgical outcomes of isolated hemorrhagic mild traumatic brain injury. Cureus. 2019, 11:e5982. 10.7759/cureus.5982

29. Marincowitz C, Lecky FE, Townend W, Borakati A, Fabbri A, Sheldon TA: The risk of deterioration in GCS13-15 patients with traumatic brain injury identified by computed tomography imaging: a systematic review and meta-analysis. J Neurotrauma. 2018, 35:703-718. 10.1089/neu.2017.5259

30. Carlson AP, Ramirez P, Kennedy G, McLean AR, Murray-Krezan C, Stippler M: Low rate of delayed deterioration requiring surgical treatment in patients transferred to a tertiary care center for mild traumatic brain injury. Neurosurg Focus. 2010, 29:E3. 10.3171/2010.8.FOCUS10182

31. Karibe H, Hayashi T, Narisawa A, Kameyama M, Nakagawa A, Tominaga T: Clinical characteristics and outcome in elderly patients with traumatic brain injury: for establishment of management strategy. Neurol Med Chir (Tokyo). 2017, 57:418-425. 10.2176/nmc.st.2017-0058

32. Takayama W, Endo A, Koguchi H, Murata K, Otomo Y: Age-related differences in the impact of coagulopathy in patients with isolated traumatic brain injury: an observational cohort study. J Trauma Acute Care Surg. 2020, 89:523-528. 10.1097/TA.0000000000002796

33. Fakhry SM, Morse JL, Garland JM, Wilson NY, Shen Y, Wyse RJ, Watts DD: Antiplatelet and anticoagulant agents have minimal impact on traumatic brain injury incidence, surgery, and mortality in geriatric ground level falls: a multi-institutional analysis of 33,710 patients. J Trauma Acute Care Surg. 2021, 90:215-223. 10.1097/TA.0000000000002985

34. Nassiri F, Badhiwala JH, Witiw CD, et al.: The clinical significance of isolated traumatic subarachnoid hemorrhage in mild traumatic brain injury: a meta-analysis. J Trauma Acute Care Surg. 2017, 83:725-731. 10.1097/TA.0000000000001617

35. Ditty BJ, Omar NB, Foreman PM, Patel DM, Pritchard PR, Okor MO: The nonsurgical nature of patients with subarachnoid or intraparenchymal hemorrhage associated with mild traumatic brain injury. J Neurosurg. 2015, 123:649-653. 10.3171/2014.10.JNS132713

36. Almenawer SA, Bogza I, Yarascavitch B, Sne N, Farrokhyar F, Murty N, Reddy K: The value of scheduled repeat cranial computed tomography after mild head injury: single-center series and meta-analysis. Neurosurgery. 2013, 72:56-62. 10.1227/NEU.0b013e318276f899

37. Fortuna GR, Mueller EW, James LE, Shutter LA, Butler KL: The impact of preinjury antiplatelet and anticoagulant pharmacotherapy on outcomes in elderly patients with hemorrhagic brain injury. Surgery. 2008, 144:598-603. 10.1016/j.surg.2008.06.009

38. Jehan F, Zeeshan M, Kulvatunyou N, et al.: Is there a need for platelet transfusion after traumatic brain injury in patients on P2Y12 inhibitors?. J Surg Res. 2019, 236:224-229. 10.1016/j.jss.2018.11.050

39. Prexl O, Bruckbauer M, Voelckel W, Grottke O, Ponschab M, Maegele M, Schöchl H: The impact of direct oral anticoagulants in traumatic brain injury patients greater than 60-years-old. Scand J Trauma Resusc Emerg Med. 2018, 26:20. 10.1186/s13049-018-0487-0

40. Greuter L, Ullmann M, Mariani L, Guzman R, Soleman J: Effect of preoperative antiplatelet or anticoagulation therapy on hemorrhagic complications in patients with traumatic brain injury undergoing craniotomy or craniectomy. Neurosurg Focus. 2019, 47:E3. 10.3171/2019.8.FOCUS19546

41. Stippler M, Keith S, Nelton EB, et al.: Pathway-based reduction of repeat head computed tomography for patients with complicated mild traumatic brain injury: implementation and outcomes. Neurosurgery. 2021, 88:773-778. 10.1093/neuros/nyaa504 


\section{Cureus}

42. Yun BJ, Borczuk P, Wang L, Dorner S, White BA, Raja AS: Evaluation of a low-risk mild traumatic brain injury and intracranial hemorrhage emergency department observation protocol. Acad Emerg Med. 2018, 25:769-775. 10.1111/acem.13350

43. Ratcliff JJ, Adeoye O, Lindsell CJ, et al.: ED disposition of the Glasgow Coma Scale 13 to 15 traumatic brain injury patient: analysis of the Transforming Research and Clinical Knowledge in TBI study. Am J Emerg Med. 2014, 32:844-850. 10.1016/j.ajem.2014.04.003

44. Joseph B, Pandit V, Haider AA, et al.: Improving hospital quality and costs in nonoperative traumatic brain injury: the role of acute care surgeons. JAMA Surg. 2015, 150:866-872. 10.1001/jamasurg.2015.1134

45. Ullman JS, Timmons SD, Valadka AB: Neurosurgeons' critical role in managing traumatic brain injury . JAMA Surg. 2016, 151:199-200. 10.1001/jamasurg.2015.3642

46. Manivannan S, Sharouf F, Mayo I, et al.: Management of neurotrauma during COVID-19: a single centre experience and lessons for the future. Brain Inj. 2021, 35:957-963. 10.1080/02699052.2021.1934731

47. Mao L, Jin H, Wang M, et al.: Neurologic manifestations of hospitalized patients with coronavirus disease 2019 in Wuhan, China. JAMA Neurol. 2020, 77:683-690. 10.1001/jamaneurol.2020.1127

48. Burke JF, Chan AK, Mummaneni V, et al.: Letter: the coronavirus disease 2019 global pandemic: a neurosurgical treatment algorithm. Neurosurgery. 2020, 87:E50-E56. 10.1093/neuros/nyaa116

49. Acute care neurosurgery: an evolving practice model - neurotrauma \& critical care section . (2020). Accessed: August 18, 2020: https://newsletters.aans.org/neurotrauma/spring-2020/acute-careneurosurgery-an-evolving-practice-model/. 